\title{
Targeted blockade of JAK/STAT3 signaling inhibits proliferation, migration and collagen production as well as inducing the apoptosis of hepatic stellate cells
}

\author{
YUAN-JING GU, WU-YI SUN, SEN ZHANG, XIN-RAN LI and WEI WEI
}

\begin{abstract}
Institute of Clinical Pharmacology, Anhui Medical University, Key Laboratory of Anti-inflammatory and Immune Medicine, Ministry of Education, Anhui Collaborative Innovation Center of Anti-inflammatory

and Immune Medicine, Hefei, Anhui 230032, P.R. China
\end{abstract}

Received November 5, 2015; Accepted July 8, 2016

DOI: $10.3892 / \mathrm{ijmm} .2016 .2692$

\begin{abstract}
Protein tyrosine kinases belonging to the Janus kinase (JAK) family are associated with many cytokine receptors, which, on ligand binding, regulate important cellular functions such as proliferation, apoptosis and differentiation. The protective effects of JAK inhibitors on fibrotic diseases such as myelofibrosis and bone marrow fibrosis have been demonstrated in previous studies. The JAK inhibitor SHR0302 is a synthetic molecule that potently inhibits all members of the JAK family, particularly JAK1. However, its effect on hepatic fibrosis has not been investigated to date, to the best of our knowledge. In the present study, the effects of SHR0302 on the activation, proliferation, migration and apoptosis of hepatic stellate cells (HSCs) as well as HSC collagen production were investigated. Our data demonstrated that treatment with SHR0302 $\left(10^{-9}-10^{-5} \mathrm{~mol} / \mathrm{l}\right)$ exerted an inhibitory effect on the activation, proliferation and migration of HSCs. In addition, the expression of collagen I and collagen III were significantly decreased following treatment with SHR0302. Furthermore, SHR0302 induced the apoptosis of HSCs, which was demonstrated by Annexin V/PI staining. SHR0302 significantly increased the activation of caspase-3 and Bax in HSCs whereas it decreased the expression of Bcl-2. SHR0302 also inhibited the activation of Akt signaling pathway. The pharmacological inhibition of the JAK1/signal transducer and activator of transcription (STAT)3 pathway led to the disruption of functions essential for HSC growth. Taken together, these findings provide evidence that SHR0302 may have the potential to alleviate hepatic fibrosis by targeting HSC functions.
\end{abstract}

Correspondence to: Professor Wu-Yi Sun or Professor Wei Wei, Institute of Clinical Pharmacology, Anhui Medical University, Key Laboratory of Anti-inflammatory and Immune Medicine, Ministry of Education, Anhui Collaborative Innovation Center of Anti-inflammatory and Immune Medicine, Anhui Medical University, 81 Meishan Road, Hefei, Anhui 230032, P.R. China

E-mail: sunwuyi51@aliyun.com

E-mail:wwei@ahmu.edu.cn

Key words: liver fibrosis, hepatic stellate cells, Janus kinase, signal transducer and activator of transcription, proliferation, apoptosis

\section{Introduction}

Hepatic fibrosis is the final consequence of many chronic liver injuries that later develop in cirrhosis and hepatocellular carcinoma (HCC), which are leading causes of morbidity and mortality worldwide (1). Regardless of the cause, hepatic fibrosis is always characterized by the abnormal accumulation of extracellular matrix (ECM). Accumulating evidence has showed that in liver injury, hepatic stellate cells (HSCs) undergo a phenotypic transformation from quiescent, non-proliferating, retinoid-storing cells to a proliferating, matrix-producing phenotype similar to myofibroblasts (MFBs) (2-4). Thus, the factors that regulate the activation, proliferation and functions of HSCs represent important antifibrotic targets (5). Clinical reports suggest that advanced hepatic fibrosis is potentially reversible (6); however, therapeutic options are limited. Therefore, there is an urgent need for novel effective agents capable of inhibiting the function of HSCs.

The Janus kinases (JAKs) are a family of intracellular tyrosine kinases that play essential roles in the signaling of numerous cytokines that have been implicated in the pathogenesis of many diseases. The family of JAKs comprises four members in mammals: JAK1, JAK2, JAK3 and tyrosine kinase 2 (Tyk2) (7). After the engagement of cytokine receptors constitutively bound to JAK, JAK is activated by a conformational change and phosphorylated. This in turn phosphorylates the cytokine receptors, resulting in the phosphorylation of signal transducer and activator of transcription (STAT) that subsequently translocates into the nucleus, in order to regulate gene expression (8). The JAK/STAT pathway mediates a plethora of cellular functions including defense against pathogens, differentiation, proliferation, apoptosis, metabolism and cellular transformation $(7,9)$. Some JAK1/2 inhibitors have been demonstrated to exert protective effects in fibrotic diseases, such as bone marrow fibrosis and myelofibrosis (10). However, the therapeutic effect of JAK inhibition in hepatic fibrosis has not been investigated to date, to the best of our knowledge. The JAK inhibitor SHR0302 $\left(\mathrm{C}_{18} \mathrm{H}_{22} \mathrm{~N}_{8} \mathrm{O}_{2} \mathrm{~S} \cdot \mathrm{H}_{2} \mathrm{SO}_{4}, \mathrm{MW}, 512\right)$, binds to JAK1 with a stronger affinity than to other JAKs (selectivity for JAK1 is $>10$ times for JAK2, 77 times for JAK3 and 420 times for Tyk2). This study examined the effects of 
the JAK inhibitor SHR0302 on the activation, proliferation, migration, collagen production and apoptosis of HSCs and the underlying mechanisms responsible for these effects. Our findings demonstrated the protective effects of SHR0302 on hepatic fibrosis by inhibiting HSC functions.

\section{Materials and methods}

Chemicals and reagents. SHR0302 was obtained from Jiangsu Hengrui Medicine Co., Ltd. (Jiangsu, China). MTT was purchased from Sigma Chemical Co. (St. Louis, MO, USA). The cell apoptosis Annexin V/PI detection kit was obtained from Shanghai Bestbio (Shanghai, China). Transforming growth factor $\beta 1$ (TGF- $\beta 1$ ) was obtained from Peprotech EC, Ltd. (London, UK). Phosphorylated (p-)STAT3 (9145), STAT3 (4904), p-Akt (4058) and Akt (4691) primary antibodies were purchased from Cell Signaling Technology (Beverly, MA, USA). p-JAK1, JAK, cleaved caspase-3 (sc-98785), $\alpha$-smooth muscle actin ( $\alpha$-SMA; sc-53015), collagen I (sc-59772) and collagen III (sc-8780-R) primary antibodies were purchased from Santa Cruz Biotechnology (Santa Cruz, CA, USA). Bcl-2 (TA803003), Bax (TA346891) and $\beta$-actin (TA-09) primary antibodies were purchased from ZSGB-Bio (Beijing,China). Dulbecco's modified Eagle's medium (DMEM) and fetal bovine serum (FBS) were obtained from Gibco (Carlsbad, CA, USA). Other chemicals used in the experiment were of analytical grade and obtained from commercial sources.

Cell culture. The HSC-T6 cell line was obtained from the Institute of Liver Disease at the Shanghai University of Traditional Chinese Medicine (Shanghai, China). The HSCs were cultured at $37^{\circ} \mathrm{C}$ in an atmosphere of $5 \% \mathrm{CO}_{2}$ in DMEM containing $10 \%$ FBS, $2 \mathrm{mmol} / \mathrm{l} \mathrm{L}$-glutamine, $100 \mathrm{U} / \mathrm{ml}$ of penicillin and $100 \mu \mathrm{g} / \mathrm{ml}$ of streptomycin.

Cell proliferation assay. The MTT assay was used to evaluate the proliferation of HSCs. Briefly, the HSCs were plated at a density of $5 \times 10^{4}$ cells $/ \mathrm{ml}$ in 96 -well culture plates. The confluent cells were growth-arrested in DMEM containing $0.5 \% \mathrm{FBS}$ for $24 \mathrm{~h}$. Subsequently, the cells were incubated with SHR0302 at various concentrations $\left(10^{-9}, 10^{-8}, 10^{-7}, 10^{-6}\right.$ and $\left.10^{-5} \mathrm{~mol} / \mathrm{l}\right)$ for $48 \mathrm{~h}$. Following treatment, MTT solution $(5.0 \mathrm{mg} / \mathrm{ml}$ in PBS) was added $(20.0 \mu \mathrm{l} / \mathrm{well})$, and the plates were incubated at $37^{\circ} \mathrm{C}$ in $5 \% \mathrm{CO}_{2}$ for a further $4 \mathrm{~h}$. The MTT-formazan product was dissolved in $150 \mu \mathrm{l}$ dimethyl sulfoxide (DMSO)/well. After $10 \mathrm{~min}$, the plates were read on a BioTek Elx808 microplate reader (Winooski, VT, USA) at $570 \mathrm{~nm}$.

Migration assay. To examine the effects of SHR0302 on the migration of HSCs, a wound-healing assay was performed. The HSCs were seeded in 6-well plates at 80-90\% confluency. The cell monolayer was then wounded with a $200 \mu \mathrm{l}$-pipette. After washing with PBS three times to remove cell debris, the cells were incubated with $5 \mathrm{ng} / \mathrm{ml}$ TGF- $\beta 1$ and SHR0302 at various concentrations $\left(10^{-9}, 10^{-8}, 10^{-7}, 10^{-6}\right.$ and $\left.10^{-5} \mathrm{~mol} / \mathrm{l}\right)$ for $24 \mathrm{~h}$, and then images were captured under a microscope (IX71; Olympus, Tokyo, Japan) at 0 and $24 \mathrm{~h}$ after treatment. Cell migration was determined by measuring the movement of cells into the scraped area and quantitative analysis showing the percentage wound closure relative to different conditioned media from the HSCs was performed using Adobe Photoshop Elements 6.0 software.

Flow cytometric analysis. The HSCs were plated in 6-well plates and treated with SHR0302 $\left(10^{-9}, 10^{-8}, 10^{-7}, 10^{-6}\right.$ and $\left.10^{-5} \mathrm{~mol} / \mathrm{l}\right)$ for $48 \mathrm{~h}$. After being harvested by trypsinization, the cells were washed twice with cold PBS, and $400 \mu 1 \mathrm{XX}$ binding buffer was added to each sample tube at a density of $1.0 \times 10^{6} \mathrm{cells} / \mathrm{ml}$. The sample solutions were then transferred to a $5 \mathrm{ml}$ culture tube and incubated with $5 \mu \mathrm{l}$ FITC-conjugated Annexin V for $15 \mathrm{~min}$ at $2-8^{\circ} \mathrm{C}$ in the dark, and $10 \mu \mathrm{PI}$ for $5 \mathrm{~min}$ at $2-8^{\circ} \mathrm{C}$ in the dark. The cells were collected and apoptosis was examined using a flow cytometer (FC 500; Beckman Coulter, Inc., Brea, CA, USA).

Western blot analysis. Proteins were then extracted from the cells in RIPA lysis buffer [50 mmol/1 Tris-HCl, $\mathrm{pH} 7.4$, $150 \mathrm{mmol} / 1 \mathrm{NaCl}, 10 \mathrm{mmol} / 1$ phenylmethylsulfonyl fluoride(PMSF), $1 \mathrm{mmol} /$ lethylenediaminetetraacetic acid(EDTA), $0.1 \%$ sodium dodecyl sulfate (SDS), $1 \%$ Triton $\mathrm{X}-100,1 \%$ sodium deoxycholate]. The protein concentration was determined using the Bradford assay. A protein sample was mixed with the 5X sample buffer (4:1) (Bio-Rad Laboratories, Inc., Hercules, CA, USA) and heated in boiling water for $10 \mathrm{~min}$. The proteins were resolved by SDS-PAGE, transferred to a polyvinylidene fluoride (PVDF) membrane (Millipore, Bedford, MA, USA). After blocking with 5\% non-fat milk (blocking solution) at room temperature, the membrane was incubated with $\alpha$-SMA, collagen I, collagen III, cleaved caspase-3, Bcl-2, Bax, p-JAK1, JAK1, p-STAT3, STAT3, p-Akt, Akt and $\beta$-actin primary antibodies $(1: 1,000)$ overnight at $4^{\circ} \mathrm{C}$. After washing the blot in TBST three times, horseradish peroxidase (HRP)-conjugated secondary antibodies were applied for $2 \mathrm{~h}$ at room temperature. After extensive washing in TBST, immunodetection was visualized by enhanced chemiluminescence (Pierce, Rockford, IL, USA) using hydrogen peroxide and luminol as substrates. Autoradiographs were scanned using ImageQuant LAS 4000 mini (GE Healthcare Bio-Sciences AB, Uppsala, Sweden). The density of the specific bands was quantified using ImageJ software (National Institutes of Health, Bethesda, MD, USA).

Statistical analysis. Data are expressed as the means \pm SD and statistical analysis was performed using one-way ANOVA. All statistical analysis was performed with the statistical package SPSS 13.0 (SPSS, Inc., Chicago, IL, USA). P $<0.05$ was considered to indicate a statistically significant difference.

\section{Results}

SHR0302 suppresses the activation of HSCs. Since $\alpha$-SMA is an marker of activated HSCs (11), we investigated the activity of HSCs by examining the expression of $\alpha$-SMA. As illustrated in Fig. 1, SHR0302 at various concentrations markedly inhibited the expression of $\alpha$-SMA compared with the untreated group. These results suggest that SHR0302 inhibits the activation of HSCs by suppressing the expression of $\alpha$-SMA.

SHR0302 inhibits the proliferation of HSCs. The effect of SHR0302 on the proliferation of HSCs was detected using the MTT assay. HSCs were incubated with SHR0302 at various concentrations $\left(10^{-9}, 10^{-8}, 10^{-7}, 10^{-6}\right.$ and $\left.10^{-5} \mathrm{~mol} / \mathrm{l}\right)$. As shown 
Table I. Effect of SHR0302 on the proliferation of HSCs (means \pm SD, n=8).

\begin{tabular}{lccc}
\hline & & & \\
\cline { 3 - 4 } Group & $\begin{array}{c}\text { Concentration } \\
(\mathrm{mol} / \mathrm{l})\end{array}$ & $\mathrm{A}_{570 \mathrm{~nm}}$ & $\mathrm{~h}$ \\
\hline Control & - & $0.782 \pm 0.060$ & - \\
SHR0302 & $10^{-9}$ & $0.733 \pm 0.055$ & 6.376 \\
& $10^{-8}$ & $0.730 \pm 0.056$ & 6.650 \\
& $10^{-7}$ & $0.669 \pm 0.080^{\mathrm{a}}$ & 14.551 \\
& $10^{-6}$ & $0.632 \pm 0.025^{\mathrm{a}}$ & 19.143 \\
& $10^{-5}$ & $0.402 \pm 0.046^{\mathrm{a}}$ & 48.640 \\
\hline
\end{tabular}

${ }^{\mathrm{a}} \mathrm{P}<0.01$ compared with control group. HSC, hepatic stellate cell.

A

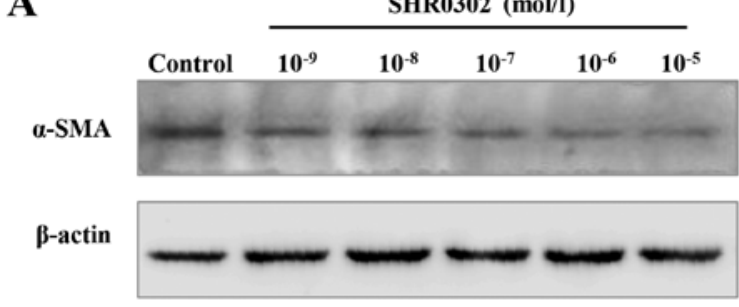

B

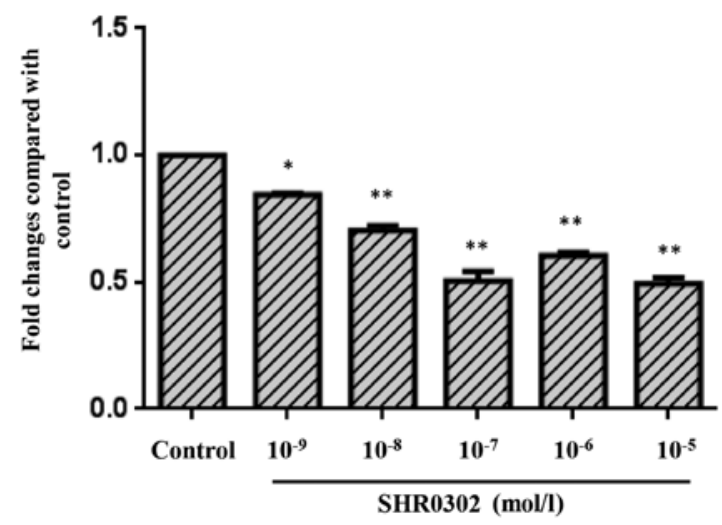

Figure 1. Effect of SHR0302 on the expression of $\alpha$-smooth muscle actin ( $\alpha$-SMA) in hepatic stellate cells (HSCs). (A) Western blots of $\alpha$-SMA expression in HSCs cultured with SHR0302 at the various concentrations $\left(10^{-9}, 10^{-8}\right.$, $10^{-7}, 10^{-6}$ and $10^{-5} \mathrm{~mol} / \mathrm{l}$ ) for $24 \mathrm{~h}$. HSCs without SHR0302 treatment were used as the control. (B) The semi-quantitative analysis of $\alpha$-SMA expression in the conditioned media from HSCs. The data are expressed as the means \pm SD in the three independent experiments. The band intensity of $\alpha$-SMA was quantified by densitometry and normalized to $\beta$-actin. ${ }^{*} \mathrm{P}<0.05$, ${ }^{* *} \mathrm{P}<0.01$ compared with the control group.

in Table I, the inhibitory rates of SHR0302 $\left(10^{-9}, 10^{-8}, 10^{-7}\right.$, $10^{-6}$ and $10^{-5} \mathrm{~mol} / \mathrm{l}$ ) in HSCs were 6.376, 6.650, 14.551, 19.143 and $48.640 \%$, respectively, following treatment for $48 \mathrm{~h}$. The MTT experiment confirmed that HSCs were sensitive to SHR0302, that SHR0302 displayed an inhibitory effect on the proliferation of HSCs, and that inhibition ocurred in a concentration-dependent manner.

SHR0302 inhibits the migration of HSCs. To determine the effect of SHR0302 on the migration of HSCs, wound-healing assays was performed after exposing cells to different concentrations of SHR0302 in the presence or absence of TGF- $\beta 1$. As shown in Fig. 2, TGF- $\beta 1$ markedly enhanced cell migration during the wound-healing process compared with the control group. By contrast, SHR0302 effectively attenuated the migration ability of HSCs at concentrations of $10^{-7}, 10^{-6}$ and $10^{-5} \mathrm{~mol} / \mathrm{l}$, which shows that SHR0302 directly inhibited the migration of HSCs.

SHR0302 decreases the expression of collagen I and collagen III in HSCs. To determine the effect of JAK inhibitor SHR0302 on collagen I and collagen III synthesis in HSCs, western blot analysis was performed. TGF- $\beta 1$ strongly promotes HSCs to synthesize ECM, such as collagen and fibronetin $(12,13)$. The control groups showed low levels of collagen I and collagen III whereas treatment with TGF- $\beta 1$ significantly increased the expression of collagen I and collagen III in HSCs. SHR0302 treatment at concentrations of $10^{-8}, 10^{-7}, 10^{-6}$ and $10^{-5} \mathrm{~mol} / \mathrm{l}$ markedly reduced the production of both collagen I and collagen III in HSCs (Fig. 3). Our data therefore demonstrated that SHR0302 reduces collagen deposition.

SHR0302 induces the apoptosis of HSCs. To determine whether SHR0302 induced the apoptosis of HSCs, the apoptosis rates were detected by Annexin V/PI staining. As shown in Fig. 4, the percentage of apoptotic cells increased from $6.76 \%$ in the control group to $75.45 \%$ in the SHR0302 $10^{-5} \mathrm{~mol} / \mathrm{l}$ treatment group of HSCs after $48 \mathrm{~h}$. These results showed that SHR0302 significantly induced the apoptosis of HSCs.

SHR0302 modulates the expression of Bcl-2 and Bax in HSCs. To identify a possible mechanism by which SHR0302 induced the apoptosis of HSCs, western blot analysis was performed (Fig. 5A). The results of western blot analysis revealed the decreased expression of Bcl-2 in the SHR0302-treated HSCs compared with the control group. SHR0302 at various concentrations markedly inhibited Bcl-2 expression. However, the expression of Bax was elevated at various concentrations of SHR0302-treated cells compared with the control group. As shown in Fig. 5B, the Bax to Bcl-2 ratio was significantly increased in the SHR0302-treated cells compared with the control group. The results indicated that SHR0302 induced the apoptosis of HSCs mainly by regulating the expression of $\mathrm{Bcl}-2$ family proteins. 
A
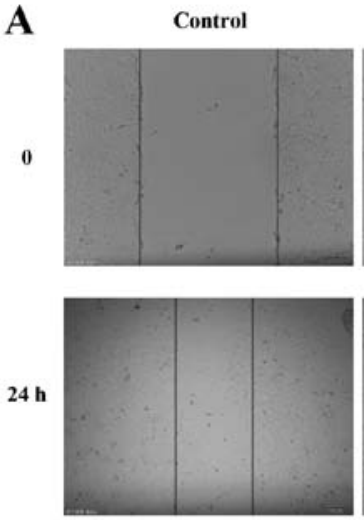

SHR0302 $\left(10^{-6} \mathrm{~mol} / \mathrm{l}\right)+\mathrm{TGF}-\beta 1$
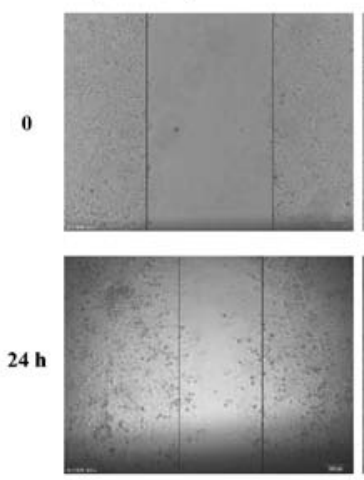

TGF- $\beta 1$
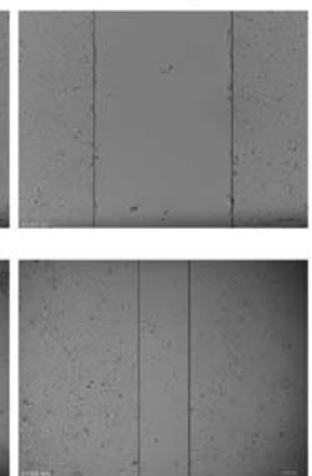

SHR0302

$\left(10^{-5} \mathrm{~mol} / \mathrm{l}\right)+\mathrm{TGF}-\beta 1$
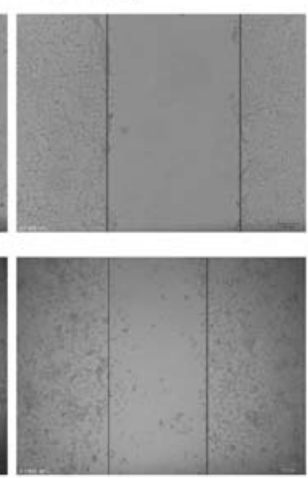
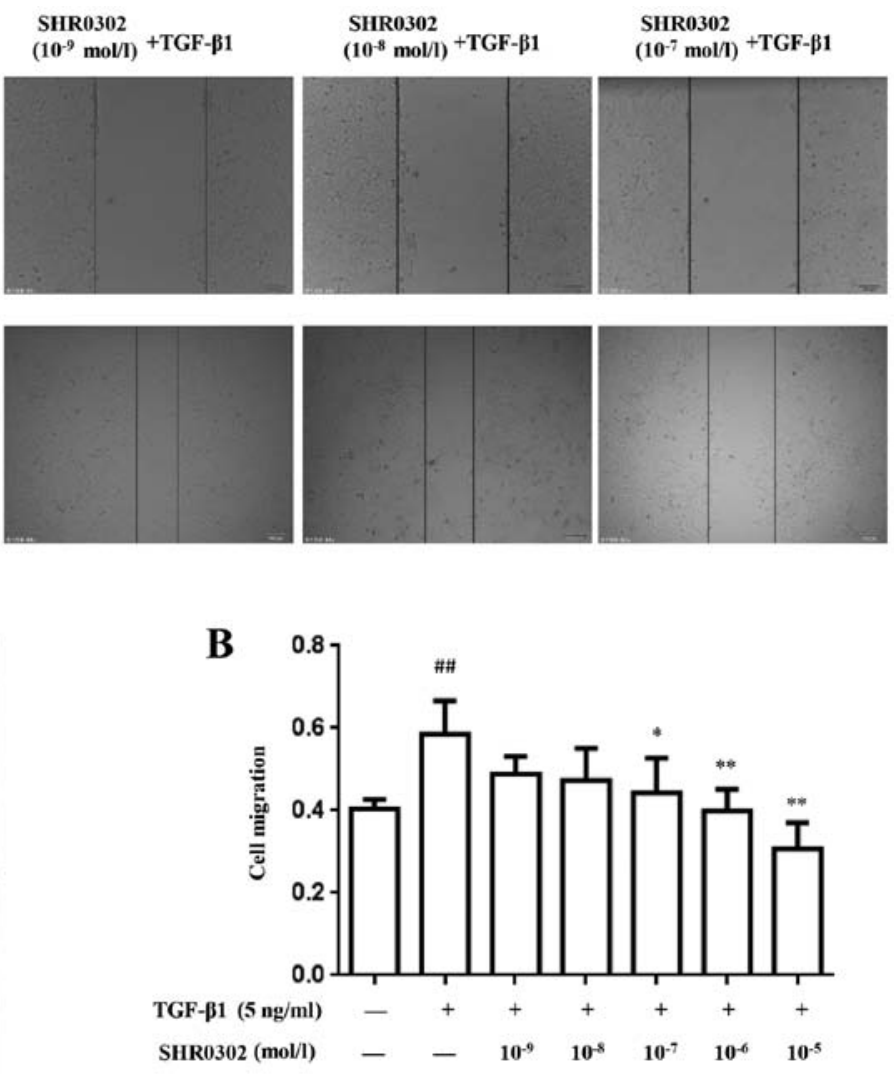

Figure 2. Effect of SHR0302 on the migration of hepatic stellate cells (HSCs). (A) Images of HSCs following treatment with SHR0302 at concentrations of $10^{-9}$, $10^{-8}, 10^{-7}, 10^{-6}$ and $10^{-5} \mathrm{~mol} / 1$ at 0 and $24 \mathrm{~h}$ in TGF- $\beta 1$-stimulated HSCs. HSCs without TGF- $\beta 1$ treatment were used as the control group. (B) Quantitative analysis showed the percentage wound closure relative to different conditioned media from HSCs using Adobe Photoshop Elements 6.0 software. The data are expressed as the means $\pm \mathrm{SD}$ in the three independent experiments. ${ }^{\# \#} \mathrm{P}<0.01$ compared with the control group, ${ }^{*} \mathrm{P}<0.05,{ }^{* *} \mathrm{P}<0.01$ compared with the TGF- $\beta 1$ group.

A

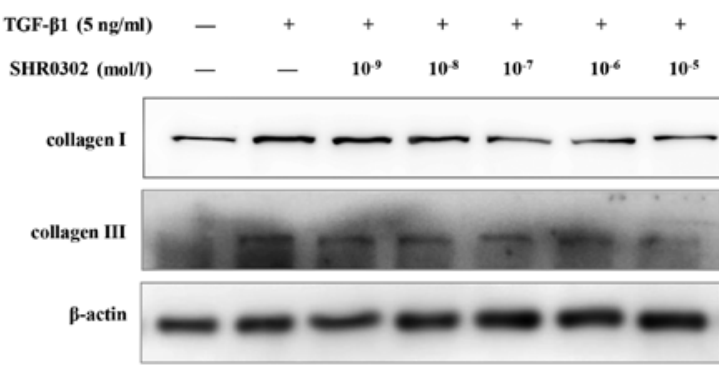

B

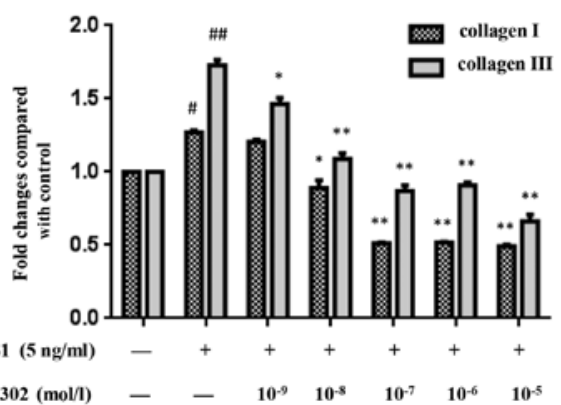

Figure 3. Effects of SHR0302 on collagen I and collagen III expression in hepatic stellate cells (HSCs). (A) Western blots of collagen I and collagen III expression in TGF- $\beta 1$-stimulated HSCs treated with various concentrations of SHR0302 $\left(10^{-9}, 10^{-8}, 10^{-7}, 10^{-6}\right.$ and $\left.10^{-5} \mathrm{~mol} / \mathrm{l}\right)$. (B) The semi-quantitative analysis of collagen I and collagen III expression in the conditioned media from HSCs. The data are expressed as the means \pm SD from three independent experiments. The band intensity of collagen I and collagen III were quantified by densitometry and normalized to $\beta$-actin. ${ }^{\#} \mathrm{P}<0.05,{ }^{\# \prime} \mathrm{P}<0.01$ compared with the control group, ${ }^{*} \mathrm{P}<0.05,{ }^{* *} \mathrm{P}<0.01$ compared with the TGF- $\beta 1$ group.
SHR0302 increases caspase- 3 activation in HSCs. To determine whether SHR0302 affects the activation of caspase-3 in HSCs, the activation of caspase- 3 was quantified through western blot analysis. As shown in Fig. 6, a low level of cleaved caspase-3 was observed in the control group. Treatment with various concentrations of SHR0302 markedly increased the levels of cleaved caspase-3 in HSCs.

SHR0302 suppresses the JAK1/STAT 3 signaling pathway in HSCs. The pathogenesis of hepatic fibrosis is closely associated with the activation of STAT3 (14). SHR0302 resulted in dose-dependent decreases in JAK1 and STAT3 phosphorylation (Fig. 7). Also, similar concentrations of SHR0302 exerted effects on the activation, proliferation, collagen deposition and apoptosis of HSCs. These findings suggest that the mechanism responsible for the effects of SHR0302 on HSC function occurs through the inhibition of JAK1/STAT3 signaling pathways.

SHR0302 suppresses the Akt signaling pathway in HSCs. The Akt signaling pathway is a major regulator of crucial cell functions such as cell growth, survival and proliferation. The inhibition of Akt activation may induce the apoptosis of HSCs and suppress collagen synthesis $(15,16)$ in hepatic fibrosis. Thus, we examined whether SHR0302 has an effect on the Akt signaling pathway. The results (Fig. 8) showed that SHR0302 significantly suppressed the Akt signaling pathway at concentrations of $10^{-5}, 10^{-6}$ and $10^{-7} \mathrm{~mol} / 1$. 
A

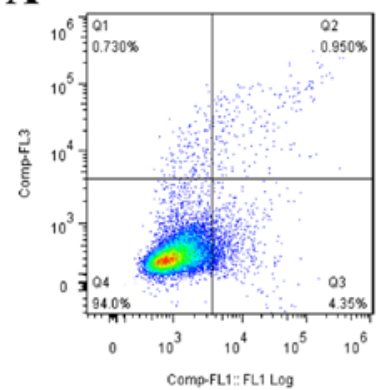

SHR0302 10-7 $(\mathrm{mol} / \mathrm{l})$

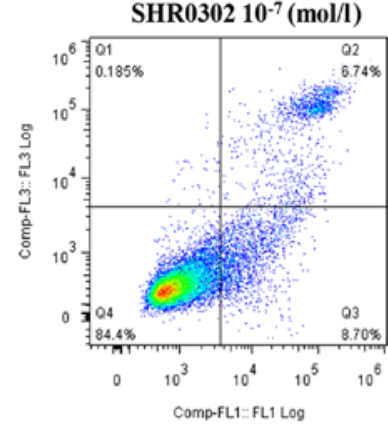

SHR0302 $10^{-9}(\mathrm{~mol} / \mathrm{l})$

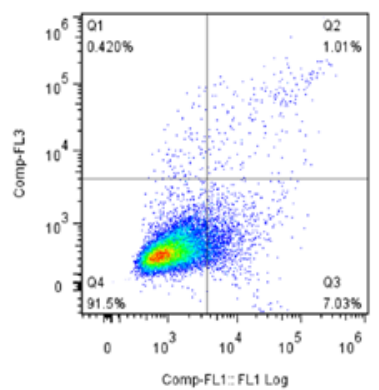

SHR0302 10-6 $(\mathrm{mol} / \mathrm{l})$

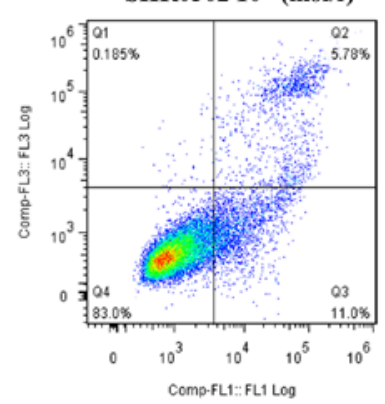

SHR0302 10-8 $(\mathrm{mol} / \mathrm{l})$

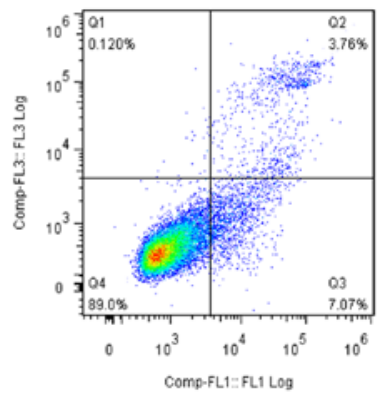

SHR0302 $10^{-5}(\mathrm{~mol} / \mathrm{l})$

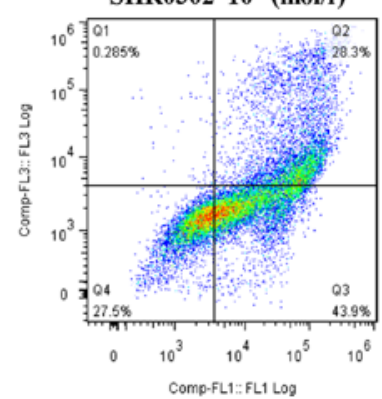

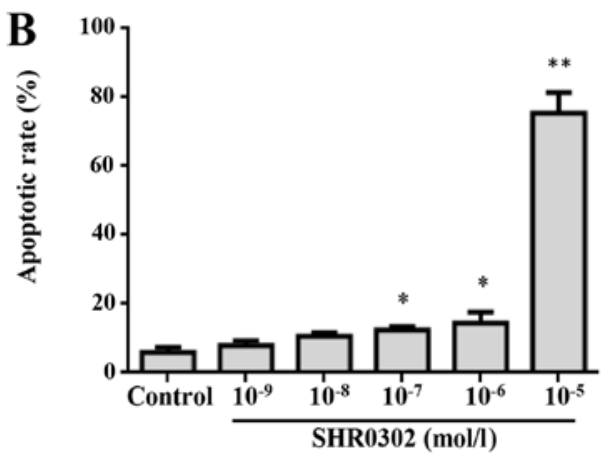

Figure 4. Effect of SHR0302 on the apoptosis of hepatic stellate cells (HSCs). (A) The representative flow cytometry dot plot of each group. HSCs without any SHR0302 treatment were used as the control group. Frames are divided into four quadrants: early apoptotic cells (Annexin $\left.\mathrm{V}^{+} / \mathrm{PI}^{-}\right)$are in quadrant III; late apoptotic cells (Annexin $\mathrm{V}^{+} / \mathrm{PI}^{+}$) are in quadrant II; normal cells (Annexin $\mathrm{V}^{-} / \mathrm{PI}^{-}$) are in quadrant IV; and necrotic cells injured by experimental manipulation (Annexin $\mathrm{V}^{-} / \mathrm{PI}^{+}$) are in quadrant I. (B) Percentages of apoptotic cells (early apoptotic cells and late apoptotic cells) in HSCs treated with SHR0302. The data are expressed as the means $\pm \mathrm{SD}$ from three independent experiments. ${ }^{*} \mathrm{P}<0.05,{ }^{* *} \mathrm{P}<0.01$ compared with the control group.

\section{Discussion}

Hepatic fibrosis is the pathological consequence of chronic liver diseases, resulting from the progressive accumulation of ECM, which is mainly enriched in types I and III fibrillar collagens. In the advanced stages, fibrosis leads to cirrhosis, a condition characterized by abnormal liver architecture, failing liver function and portal hypertension as well as a high susceptibility to infection and to developing HCC $(17,18)$. To date, the most effective therapy for the treatment of hepatic fibrosis involves removal of the causative agent. During liver injury, HSCs undergo transdifferentiation from the lipocyte phenotype to MFBs; during this process, lipid droplets decrease in size and number whereas proliferation, migration rate and ECM production are increased $(19,20)$, and this appears to be the dominant driving force in fibrosis. These studies explored the rationale for choosing HSCs as a target for pharmacological, molecular and other novel therapeutics, for potential application in the treatment of hepatic fibrosis. Our data demonstrated that the abrogation of the
JAK1/STAT3 signaling pathway, induced by JAK inhibitor SHR0302 in HSCs, SHR0302 $\left(10^{-9}-10^{-5} \mathrm{~mol} / \mathrm{l}\right)$ exerted an inhibitory effect on the activation, proliferation and migration of HSCs. Additionally, the expression of collagen I and collagen III was decreased after treatment with SHR0302. Furthermore, SHR0302 induced the apoptosis of HSCs, which may occur through the regulation of caspase- 3 and the Bcl-2 family.

Once HSCs are activated, they become $\alpha$-SMA-expressing MFBs and migrate to the site of hepatic injury. Once there, they proliferate and express various signal transduction proteins, producing both pro-inflammatory cytokines and a great deal of collagen-rich ECM $(21,22)$. In fact, the majority of antifibrotic treatments currently under evaluation are aimed at inhibiting the activation and proliferation of HSCs as well as ECM synthesis (23). For this reason, in hepatic fibrosis, $\alpha$-SMA is considered as an indicator of activated HSCs, and contributes to the proliferation and migration of HSCs as well as collagen deposition. In previous studies, the activation of renal interstitial fibroblasts was accompanied by 
A

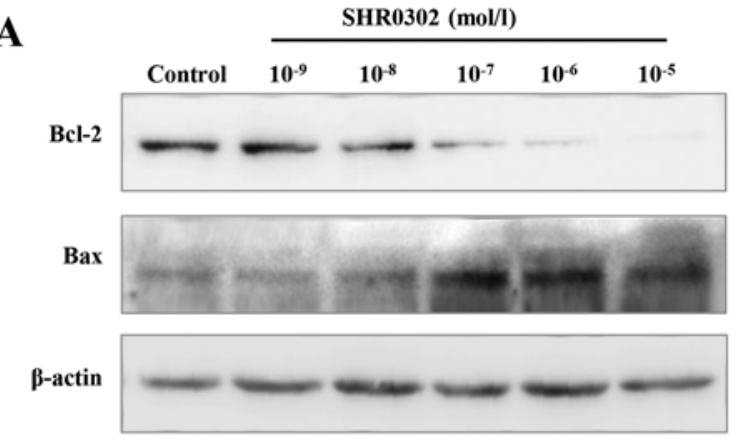

B

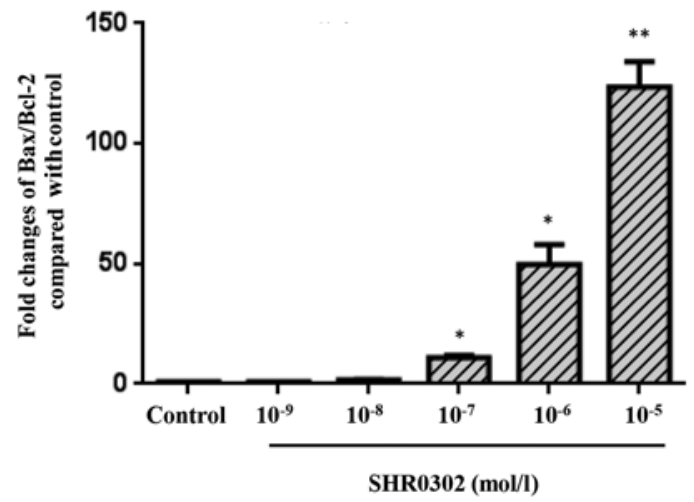

Figure 5. Effects of SHR0302 on Bcl-2 and Bax protein expression in hepatic stellate cells (HSCs). (A) Bcl-2 and Bax protein expression in the control group and the groups treated with various concentrations of SHR0302 $\left(10^{-9}\right.$, $10^{-8}, 10^{-7}, 10^{-6}$ and $\left.10^{-5} \mathrm{~mol} / \mathrm{l}\right)$. (B) The semi-quantitative analysis of Bax/Bcl-2 ratio in the conditioned media from HSCs. The data are expressed as the means \pm SD from three independent experiments. The band intensity of $\mathrm{Bcl}-2$ and Bax were quantified by the ratio of Bax to Bcl-2. ${ }^{*} \mathrm{P}<0.05,{ }^{* *} \mathrm{P}<0.01$ compared with the control group.

the phosphorylation of STAT3; the inhibition of the STAT3 pathway inhibited the expression of $\alpha$-SMA and fibronectin in a dose- and time-dependent manner (24), and suppressed collagen expression in renal fibrosis (25). Furthermore, a JAK inhibitor through STAT3 signaling reduced the leptin-mediated increase in the levels of $\alpha$-SMA and the activity of HSCs in hepatic fibrosis (26). The MTT assay in our study demonstrated that the JAK inhibitor SHR0302 significantly decreased the proliferation of HSCs. Furthermore, western blot analysis demonstrated that following treatment with SHR0302, the expression of $\alpha$-SMA was decreased in HSCs which suggests that the activation of HSCs was suppressed.

Cell migration is a vital step in the development and aggravation of several diseases, from organ fibrosis to cancer (27). The inhibition of interleukin (IL)-6-induced JAK/STAT3 phosphorylation strongly reduced the proliferation and migration of glioblastoma cells (28), and the inhibition of STAT3 expression delayed cell migration in keloid fibroblasts (29). The migration of HSCs may contribute to their accumulation at sites of liver injury. Following activation, cultured HSCs migrate in response to many stimuli. In the present study, wound-healing assays were designed to determine the migratory ability of HSCs following treatment with SHR0302. The results showed that SHR0302 significantly inhibited the migration of HSCs and provided new insights into the potential use of SHR0302 for controlling the development of hepatic fibrosis.

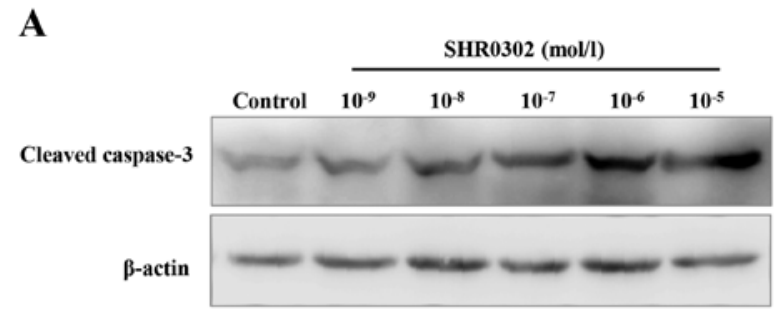

B

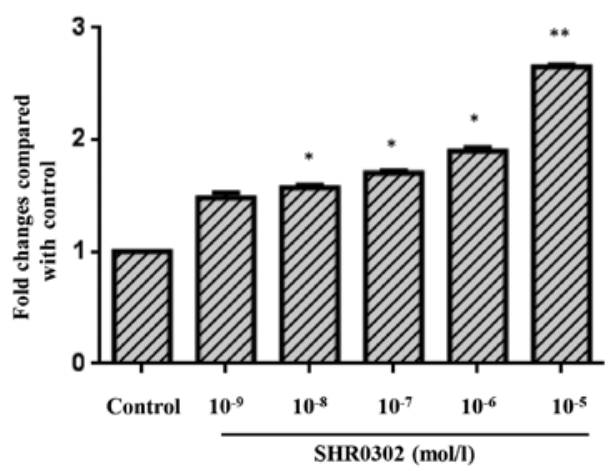

Figure 6. Effect of cleaved caspase-3 protein expression in hepatic stellate cells (HSCs). (A) Treatment of HSCs with SHR0302 $\left(10^{-9}, 10^{-8}, 10^{-7}, 10^{-6}\right.$ and $10^{-5} \mathrm{~mol} / \mathrm{l}$ ) increased the expression of cleaved caspase-3. (B) The semi-quantitative analysis of cleaved caspase- 3 in the conditioned media from HSCs. The data are expressed as the means \pm SD from three independent experiments. The band intensity of cleaved caspase- 3 was quantified by densitometry and normalized to $\beta$-actin. ${ }^{*} \mathrm{P}<0.05,{ }^{* *} \mathrm{P}<0.01$ compared with the control group.

The most striking biological consequence of the activation of HSCs is the marked increase in collagen deposition. In the human body, collagen represents about one third of the total protein, but only 5 to $10 \%$ of the protein in a normal liver. In cirrhosis, however, it amounts to $50 \%$ or more (30). The principal ECM protein products in the progression of liver fibrogenesis are predominantly collagen types I and III $(31,32)$. The blockade of STAT3 signaling inhibits collagen I in hepatic fibrosis $(26,33)$. During the development of fibrosis, HSCs respond to TGF- $\beta$ by moving to a MFB phenotype, which in turn produces a higher deposition of ECM proteins including collagen I and collagen III $(13,34)$. The results of western blot analysis revealed that TGF- $\beta 1$ induced the activation of HSCs which expressed high levels of collagen I and collagen III. Following treatment with SHR0302, type I and III collagen expression was suppressed, suggesting that SHR0302 inhibits the expression of collagen, and further decreased the deposition of ECM.

Apoptosis is a physiological process of programmed cell death that plays a vital role in maintaining tissue homeostasis (35). The apoptosis of activated HSCs is a key factor in the regression of liver fibrosis; emerging experimental and clinical evidence indicates that even cirrhosis is potentially reversible $(5,6)$. The key to this is the discovery that reversion of fibrosis is accompanied by the clearance of HSCs by apoptosis. Furthermore, proof-of-concept studies in rodents have demonstrated that the experimental augmentation of the apoptosis of HSCs promotes the regression of fibrosis $(36,37)$. Our flow cytometry assays revealed that SHR0302 significantly induces the apoptosis of HSCs. To further elucidate the possible pro-apoptotic mechanisms induced by SHR0302, 
A

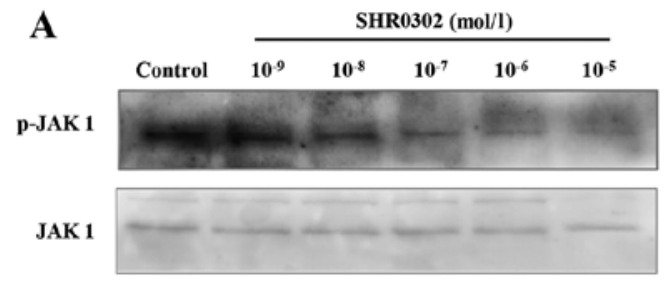

B

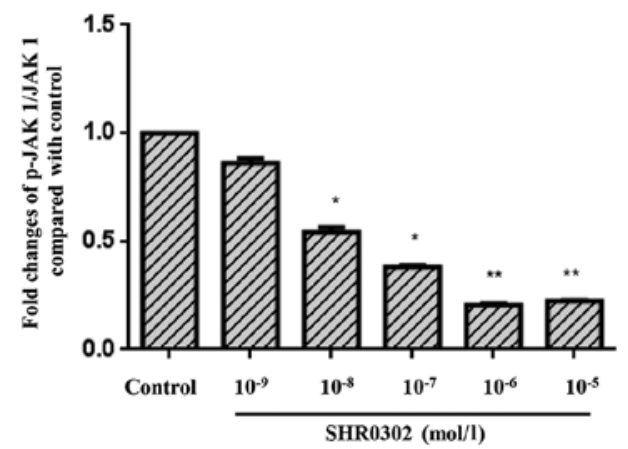

C

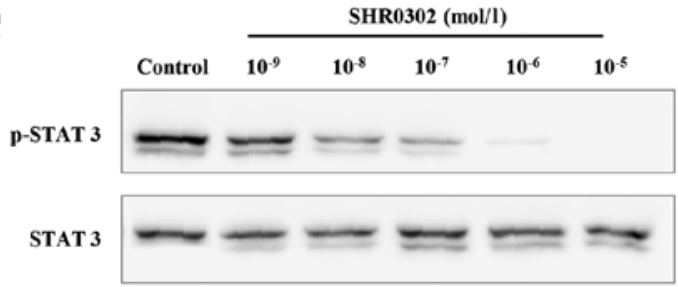

D

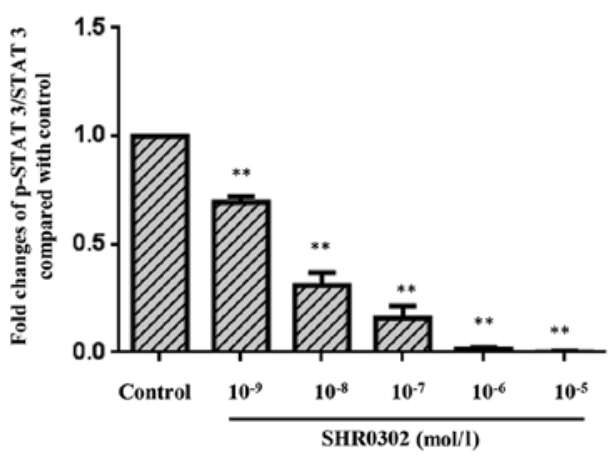

Figure 7. Effects of SHR0302 on Janus kinase (JAK)-1 and signal transducer and activator of transcription (STAT)-3 activation in HSCs. Western blots showed that SHR0302 causes the inhibition of (A) JAK1 and (C) STAT3 activation in HSCs. (B and D) The changes in phosphorylated (p-)JAK1 and p-STAT3 are expressed as ratios of phosphorylated/unphosphorylated forms and are shown as a bar diagram. The data are expressed as the means \pm SD from three independent experiments. Densitometry values in the histograms were expressed as fold change relative to the control, which was assigned a value of 1 . "P<0.05, ${ }^{* *} \mathrm{P}<0.01$ compared with the control group.

A

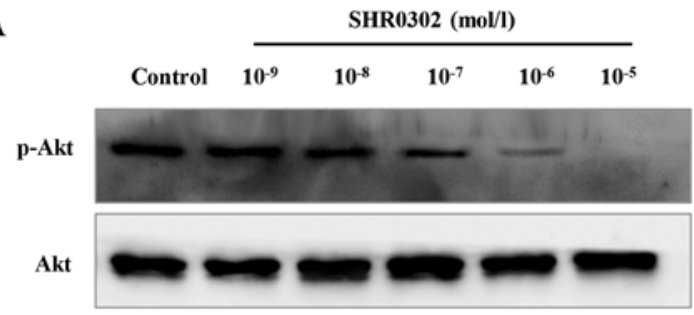

B

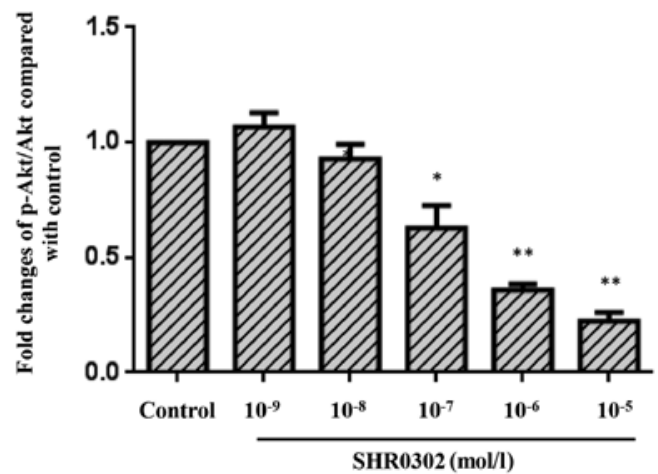

Figure 8. Effects of SHR0302 on Akt activation in hepatic stellate cells (HSCs). (A) Treatment with SHR0302 $\left(10^{-9}, 10^{-8}, 10^{-7}, 10^{-6}\right.$ and $\left.10^{-5} \mathrm{~mol} / \mathrm{l}\right)$ in HSCs inhibited the expression of phosphorylated (p-)Akt. (B) The changes in p-Akt are expressed as the ratio of phosphorylated/unphosphorylated forms and are shown as a bar diagram. The data are expressed as the means \pm SD from three independent experiments. Densitometry values in the histograms are expressed as the fold change relative to the control, which was assigned a value of $1 .{ }^{*} \mathrm{P}<0.05,{ }^{* *} \mathrm{P}<0.01$ compared with the control group.

the expression of the Bcl-2 family proteins and caspase-3, which are acknowledged apoptosis-related regulators, was examined. Activated HSCs are resistant to many pro-apoptotic stimuli, and the main survival signal is the overexpression of Bcl-2 family members $(38,39)$. The previous study showed evidence that freshly isolated HSCs possessed high levels of the pro-apoptotic molecule Bax and undetectable expression of Bcl-2, a potent inhibitor of apoptotic cell death (39). Activated HSCs/MFBs had a complete reversal in the Bcl-2/ $\mathrm{Bax}$ ratio, and $\mathrm{Bcl}-2$-silenced cells were susceptible to apoptosis. The inhibition of STAT3 is associated with decreased Bcl-2 expression and increased Bax expression in many diseases (40-42). And in our study, western blot analysis revealed the decreased expression of $\mathrm{Bcl}-2$ and elevated levels of Bax in the HSCs treated with SHR0302 compared with the control group. These results suggest that SHR0302 modulates the expression of Bcl-2 family proteins. Caspases are known for playing an important role in the execution of apoptosis, which frequently activates death proteases, catalyzing the specific cleavage of many pivotal cellular proteins (43). Caspase-3 is a frequently activated death protease; cleaved caspase- 3 expression was much less apparent in the cirrhotic livers of wild-type mice compared with those from mice receiving drug treatment $(44,45)$. JAK inhibitor-treated HSCs are positive for active caspase-3, which indicates the presence of a higher apoptotic rate (46). Western blot analysis in this study demonstrated that SHR0302 enhanced the expression of cleaved caspase-3 in HSCs. In agreement with the data regarding the Bcl-2 family of proteins, our study indicated that SHR0302 may induce apoptosis through mechanisms which modulate the Bcl-2 family proteins and caspase-3 activity.

Following the binding of cytokines to their cognate receptor, JAKs phosphorylate STAT to modulate gene expression (47). The JAK/STAT pathway is activated in response to cytokines, growth factors and hormones, mediating a plethora of cellular functions including defense against pathogens, differentiation, 
proliferation, apoptosis, metabolism and cellular transformation (9). STAT3 plays a key role in many cellular processes such as cell growth and apoptosis, and mediates the expression of a variety of genes in response to stimuli as discussed above; these broad ranging activities make JAK/STAT3 an attractive therapeutic target. Herein, we demonstrated that SHR0302 suppressed the activation of the JAK1/STAT3 signaling pathway, and may be involved in the proliferation, migration, and apoptosis of HSCs as well as HSC collagen production.

Akt signal transduction regulates ECM deposition, HSC activation and is implicated in the development and progression of hepatic fibrosis $(15,16)$. Previous findings have demonstrated that there are complex interactions between the Akt signaling pathway and the JAK signaling pathway. In study of MCF-7 human breast cancer cells and mouse embryonic fibroblasts, it was found that the activity of STAT3 regulates Akt gene expression (48). In hepatic diseases, the JAK-dependent Akt signaling pathway plays a important role in disease progression $(49,50)$. Thus, our results showing that SHR0302 inhibits the Akt signaling pathway is consistent with the findings of other studies of JAK inhibitors suggesting that they potentially inhibit the Akt signaling pathway $(51,52)$.

The effects of JAK inhibitors have been demonstrated in rheumatoid arthritis, colon cancer, lymphoblastic leukemia, myelofibrosis and so on (53-56). For example, ruxolitinib, a JAK1 and JAK2 inhibitor, in clinical trials, alleviated the burdensome manifestations of myelofibrosis, namely splenomegaly and core symptoms (57). The JAK inhibitor AG490 inhibits the leptin-stimulated mRNA expression of JAK1, JAK2 and $\alpha 1(\mathrm{I})$ collagen in HSCs (58). Thus, we hypothesized that the JAK inhibitor SHR0302 suppresses hepatic fibrosis, and herein, we proved that the selective JAK inhibitor SHR0302 suppresses certain functions in HSCs.

In conclusion, our hypothesis will require further examination. However, taken together, these findings suggest that the blockade of the JAK/ STAT3 signaling pathways significantly decreased cell function and suppressed the Akt signaling pathway in HSCs. JAKs represent an attractive target for the development of novel targeted therapies in various clinical settings including hematologic malignancies, autoimmune disease and organ transplantation. Our study has shown that SHR0302 blocks the downstream STAT3 signaling pathway by abrogating JAK1 activity, and thereby inhibits the activation, proliferation and migration of HSCs as well as collagen synthesis by HSCs. SHR0302 also induces the apoptosis of HSCs. Currently, no optimal antifibrotic drugs available for the clinical treatment of hepatic fibrosis. These results indicate that the JAK inhibitor SHR0302 may have the potential to alleviate hepatic fibrosis.

\section{Acknowledgements}

The present study was supported by grants from the National Natural Science Foundation of China (nos. 81300332, 81173075 and 81330081), the Specialized Research Fund for the Doctoral Program of Higher Education in China (no. 20113420120002), the Natural Science Foundation of the Higher Education Institutions of Anhui Province (nos. KJ2012A153 and KJ2014A119) and Anhui Provincial Natural Science Foundation (no. 1308085QH130).

\section{References}

1. Leon DA and McCambridge J: Liver cirrhosis mortality rates in Britain from 1950 to 2002: an analysis of routine data. Lancet 367: 52-56, 2006.

2. Priya S and Sudhakaran PR: Cell survival, activation and apoptosis of hepatic stellate cells: modulation by extracellular matrix proteins. Hepatol Res 38: 1221-1232, 2008.

3. Wang P, Liu T, Cong M, Wu X, Bai Y, Yin C, An W, Wang B, Jia $J$ and You $H$ : Expression of extracellular matrix genes in cultured hepatic oval cells: an origin of hepatic stellate cells through transforming growth factor beta? Liver Int 29: 575-584, 2009.

4. Moles A, Tarrats N, Morales A, Domínguez M, Bataller R, Caballería J, García-Ruiz C, Fernández-Checa JC and Marí M: Acidic sphingomyelinase controls hepatic stellate cell activation and in vivo liver fibrogenesis. Am J Pathol 177: 1214-1224, 2010.

5. Fallowfield JA: Therapeutic targets in liver fibrosis. Am J Physiol Gastrointest Liver Physiol 300: G709-G715, 2011.

6. Povero D, Busletta C, Novo E, di Bonzo LV, Cannito S, Paternostro $\mathrm{C}$ and Parola M: Liver fibrosis: a dynamic and potentially reversible process. Histol Histopathol 25: 1075-1091, 2010.

7. Seavey MM and Dobrzanski P: The many faces of Janus kinase. Biochem Pharmacol 83: 1136-1145, 2012.

8. Tanaka Y: Recent progress and perspective in JAK inhibitors for rheumatoid arthritis: from bench to bedside. J Biochem 158: 173-179, 2015.

9. Kisseleva T, Bhattacharya S, Braunstein J and Schindler CW: Signaling through the JAK/STAT pathway, recent advances and future challenges. Gene 285: 1-24, 2002.

10. Pardanani A, Vannucchi AM, Passamonti F, Cervantes F, Barbui T and Tefferi A: JAK inhibitor therapy for myelofibrosis: critical assessment of value and limitations. Leukemia 25: 218-225, 2011.

11. Johnson SJ, Hines JE and Burt AD: Phenotypic modulation of perisinusoidal cells following acute liver injury: a quantitative analysis. Int J Exp Pathol 73: 765-772, 1992.

12. Cheng K, Yang N and Mahato RI: TGF-betal gene silencing for treating liver fibrosis. Mol Pharm 6: 772-779, 2009.

13. Kanzler S, Lohse AW, Keil A, Henninger J, Dienes HP, Schirmacher P, Rose-John S, zum Büschenfelde KH and Blessing M: TGF-betal in liver fibrosis: an inducible transgenic mouse model to study liver fibrogenesis. Am J Physiol 276: G1059-G1068, 1999.

14. Mair M, Blaas L, Österreicher $\mathrm{CH}$, Casanova $\mathrm{E}$ and Eferl R: JAK-STAT signaling in hepatic fibrosis. Front Biosci (Landmark Ed) 16: 2794-2811, 2011.

15. Paik YH, Kim JK, Lee JI, Kang SH, Kim DY, An SH, Lee SJ, Lee DK, Han KH, Chon CY, et al: Celecoxib induces hepatic stellate cell apoptosis through inhibition of Akt activation and suppresses hepatic fibrosis in rats. Gut 58: 1517-1527, 2009.

16. Son G, Hines IN, Lindquist J, Schrum LW and Rippe RA: Inhibition of phosphatidylinositol 3-kinase signaling in hepatic stellate cells blocks the progression of hepatic fibrosis. Hepatology 50: 1512-1523, 2009.

17. Schuppan D and Kim YO: Evolving therapies for liver fibrosis. J Clin Invest 123: 1887-1901, 2013.

18. Mallat A and Lotersztajn S: Cellular mechanisms of tissue fibrosis. 5. Novel insights into liver fibrosis. Am J Physiol Cell Physiol 305: C789-C799, 2013.

19. Senoo H, Yoshikawa K, Morii M, Miura M, Imai K and Mezaki Y: Hepatic stellate cell (vitamin A-storing cell) and its relative-past, present and future. Cell Biol Int 34: 1247-1272, 2010.

20. Hernandez-Gea V and Friedman SL: Pathogenesis of liver fibrosis. Annu Rev Pathol 6: 425-456, 2011.

21. Choi JH, Hwang YP, Choi CY, Chung YC and Jeong HG: Anti-fibrotic effects of the anthocyanins isolated from the purple-fleshed sweet potato on hepatic fibrosis induced by dimethylnitrosamine administration in rats. Food Chem Toxicol 48: 3137-3143, 2010.

22. Clément S, Pascarella S, Conzelmann S, Gonelle-Gispert C, Guilloux K and Negro F: The hepatitis $C$ virus core protein indirectly induces alpha-smooth muscle actin expression in hepatic stellate cells via interleukin-8. J Hepatol 52: 635-643, 2010.

23. Wu J and Zern MA: Hepatic stellate cells: a target for the treatment of liver fibrosis. J Gastroenterol 35: 665-672, 2000. 
24. Pang M, Ma L, Gong R, Tolbert E, Mao H, Ponnusamy M, Chin YE, Yan H, Dworkin LD and Zhuang S: A novel STAT3 inhibitor, S3I-201, attenuates renal interstitial fibroblast activation and interstitial fibrosis in obstructive nephropathy. Kidney Int 78: 257-268, 2010.

25. Yu Y, Wang Y, Niu Y, Fu L, Chin YE and Yu C: Leukemia inhibitory factor attenuates renal fibrosis through Stat3-miR-29c. Am J Physiol Renal Physiol 309: F595-F603, 2015.

26. Zhang W, Niu M, Yan K, Zhai X, Zhou Q, Zhang L and Zhou Y: Stat3 pathway correlates with the roles of leptin in mouse liver fibrosis and sterol regulatory element binding protein-1c expression of rat hepatic stellate cells. Int J Biochem Cell Biol 45 736-744, 2013.

27. Ridley AJ, Schwartz MA, Burridge K, Firtel RA, Ginsberg MH, Borisy G, Parsons JT and Horwitz AR: Cell migration: integrating signals from front to back. Science 302: 1704-1709, 2003

28. Michaud-Levesque J, Bousquet-Gagnon $\mathrm{N}$ and Béliveau $\mathrm{R}$ : Quercetin abrogates IL-6/STAT3 signaling and inhibits glioblastoma cell line growth and migration. Exp Cell Res 318: 925-935, 2012.

29. Lim CP, Phan TT, Lim IJ and Cao X: Stat3 contributes to keloid pathogenesis via promoting collagen production, cell proliferation and migration. Oncogene 25: 5416-5425, 2006.

30. Schuppan D: Structure of the extracellular matrix in normal and fibrotic liver: collagens and glycoproteins. Semin Liver Dis 10 : $1-10,1990$.

31. Rojkind M and Martinez-Palomo A: Increase in type I and type III collagens in human alcoholic liver cirrhosis. Proc Natl Acad Sci USA 73: 539-543, 1976.

32. Grimaud JA, Druguet M, Peyrol S, Chevalier O, Herbage D and El Badrawy N: Collagen immunotyping in human liver: light and electron microscope study. J Histochem Cytochem 28: $1145-1156,1980$

33. Lakner AM, Moore CC, Gulledge AA and Schrum LW: Daily genetic profiling indicates JAK/STAT signaling promotes early hepatic stellate cell transdifferentiation. World J Gastroenterol 16 : 5047-5056, 2010

34. Hellerbrand C, Stefanovic B, Giordano F, Burchardt ER and Brenner DA: The role of TGFbeta1 in initiating hepatic stellate cell activation in vivo. J Hepatol 30: 77-87, 1999.

35. Thompson HJ, Strange R and Schedin PJ: Apoptosis in the genesis and prevention of cancer. Cancer Epidemiol Biomarkers Prev 1: 597-602, 1992.

36. Bataller R and Brenner DA: Hepatic stellate cells as a target for the treatment of liver fibrosis. Semin Liver Dis 21: 437-451, 2001

37. Zhao W, Su W, Kuang P, Zhang L, Liu J, Yin Z and Wang X: The role of hepatic stellate cells in the regulation of T-cell function and the promotion of hepatocellular carcinoma. Int J Oncol 41: 457-464, 2012

38. Kawada N: Human hepatic stellate cells are resistant to apoptosis: implications for human fibrogenic liver disease. Gut 55: 1073-1074, 2006.

39. Novo E, Marra F, Zamara E, Valfrè di Bonzo L, Monitillo L, Cannito S, Petrai I, Mazzocca A, Bonacchi A, De Franco RS, et al: Overexpression of $\mathrm{Bcl}-2$ by activated human hepatic stellate cells: resistance to apoptosis as a mechanism of progressive hepatic fibrogenesis in humans. Gut 55: 1174-1182, 2006.

40. Nielsen M, Kaestel CG, Eriksen KW, Woetmann A, Stokkedal T, Kaltoft K, Geisler C, Röpke C and Odum N: Inhibition of constitutively activated Stat 3 correlates with altered Bcl-2/Bax expression and induction of apoptosis in mycosis fungoides tumor cells. Leukemia 13: 735-738, 1999.

41. Lee SY, Kwok SK, Son HJ, Ryu JG, Kim EK, Oh HJ, Cho ML, Ju JH, Park SH and Kim HY: IL-17-mediated Bcl-2 expression regulates survival of fibroblast-like synoviocytes in rheumatoid arthritis through STAT3 activation. Arthritis Res Ther 15: R31, 2013.
42. Moodley YP, Misso NL, Scaffidi AK, Fogel-Petrovic M, McAnulty RJ, Laurent GJ, Thompson PJ and Knight DA: Inverse effects of interleukin-6 on apoptosis of fibroblasts from pulmonary fibrosis and normal lungs. Am J Respir Cell Mol Biol 29: 490-498, 2003.

43. Galluzzi L, Kepp O and Kroemer G: Caspase-3 and prostaglandins signal for tumor regrowth in cancer therapy. Oncogene 31: 2805-2808, 2012.

44. Yang B, El Nahas AM, Thomas GL, Haylor JL, Watson PF, Wagner B and Johnson TS: Caspase-3 and apoptosis in experimental chronic renal scarring. Kidney Int 60: 1765-1776, 2001.

45. Zhou TB, Qin YH, Zhou C, Lei FY, Zhao YJ, Chen J, Su LN and Huang WF: Less expression of prohibitin is associated with increased caspase-3 expression and cell apoptosis in renal interstitial fibrosis rats. Nephrology (Carlton) 17: 189-196, 2012.

46. Jiang JX, Mikami K, Venugopal S, Li Y and Török NJ: Apoptotic body engulfment by hepatic stellate cells promotes their survival by the JAK/STAT and Akt/NF-kappaB-dependent pathways. J Hepatol 51: 139-148, 2009.

47. O'Sullivan LA, Liongue C, Lewis RS, Stephenson SE and Ward AC: Cytokine receptor signaling through the Jak-Stat-Socs pathway in disease. Mol Immunol 44: 2497-2506, 2007.

48. Xu Q, Briggs J, Park S, Niu G, Kortylewski M,Zhang S, Gritsko T, Turkson J, Kay H, Semenza GL, et al: Targeting Stat3 blocks both HIF-1 and VEGF expression induced by multiple oncogenic growth signaling pathways. Oncogene 24: 5552-5560, 2005.

49. Saxena NK, Sharma D, Ding X, Lin S, Marra F, Merlin D and Anania FA: Concomitant activation of the JAK/STAT, PI3K/AKT, and ERK signaling is involved in leptin-mediated promotion of invasion and migration of hepatocellular carcinoma cells. Cancer Res 67: 2497-2507, 2007.

50. Niu L, Wang X, Li J, Huang Y, Yang Z, Chen F, Ni H, Jin Y, Lu X and Cao Q: Leptin stimulates alpha1(I) collagen expression in human hepatic stellate cells via the phosphatidylinositol 3-kinase/ Akt signalling pathway. Liver Int 27: 1265-1272, 2007.

51. Monaghan KA, Khong T, Burns CJ and Spencer A: The novel JAK inhibitor CYT387 suppresses multiple signalling pathways, prevents proliferation and induces apoptosis in phenotypically diverse myeloma cells. Leukemia 25: 1891-1899, 2011.

52. Gross ER, Hsu AK and Gross GJ: The JAK/STAT pathway is essential for opioid-induced cardioprotection: JAK2 as a mediator of STAT3, Akt, and GSK-3 beta. Am J Physiol Heart Circ Physiol 291: H827-H834, 2006.

53. Tanaka Y and Yamaoka K: JAK inhibitor tofacitinib for treating rheumatoid arthritis: from basic to clinical. Mod Rheumatol 23: 415-424, 2013

54. An HJ, Choi EK, Kim JS, Hong SW, Moon JH, Shin JS, Ha SH, Kim KP, Hong YS, Lee JL, et al: INCB018424 induces apoptotic cell death through the suppression of pJAK1 in human colon cancer cells. Neoplasma 61: 56-62, 2014.

55. Suryani S, Bracken LS, Harvey RC, Sia KC, Carol H, Chen IM, Evans K, Dietrich PA, Roberts KG, Kurmasheva RT, et al: Evaluation of the in vitro and in vivo efficacy of the JAK inhibitor AZD1480 against JAK-mutated acute lymphoblastic leukemia. Mol Cancer Ther 14: 364-374, 2015.

56. Swaim SJ: Ruxolitinib for the treatment of primary myelofibrosis. Am J Health Syst Pharm 71: 453-462, 2014.

57. Ostojic A, Vrhovac R and Verstovsek S: Ruxolitinib for the treatment of myelofibrosis: its clinical potential. Ther Clin Risk Manag 8: 95-103, 2012.

58. Cao Q, Mak KM and Lieber CS: Leptin enhances alpha1(I) collagen gene expression in LX-2 human hepatic stellate cells through JAK-mediated $\mathrm{H}_{2} \mathrm{O}_{2}$-dependent MAPK pathways. J Cell Biochem 97: 188-197, 2006. 